\title{
El significado de la histerectomía para un grupo de hombres chilenos parejas de histerectomizadas
}

\author{
O SIGNIFICADO DA HISTERECTOMIA PARA UM GRUPO DE HOMENS CHILENOS \\ PARCEIROS DE HISTERECTOMIZADAS
}

\section{THE MEANING OF HYSTERECTOMY FOR A GROUP OF CHILEAN MEN PARTNERS OF WOMEN WHO HAVE UNDERGONE HYSTERECTOMY}

\section{Alejandra Araya Gutiérrez", María-Teresa Urrutia Soto², Daniel Jara Suazo³, Sergio Silva Solovera ${ }^{4}$, María Jesús Lira Salas ${ }^{5}$, Claudia Flores Espinoza ${ }^{6}$}

\section{RESUMEN}

Se objetivó conocer el significado de la histerectomía para un grupo de hombres chilenos, parejas de mujeres histerectomizadas (HPMH). Estudio cualitativo, con entrevistas en profundidad realizadas a 15 hombres, parejas de mujeres histerectomizadas, entre Mayo y Septiembre del 2010, previa aprobación de dos Comités de Ética. Para el análisis de los datos se utilizó la perspectiva fenomenológica descrita por Giorgi, y se realizó el análisis de contenido según Krippendorff. Los criterios de Creswell fueron utilizados para evaluar la credibilidad del análisis y asegurar la validez descriptiva. Emergieron cinco dimensiones que representan aspectos únicos del significado de la histerectomía para los hombres parejas de mujeres histerectomizadas: síntomas, comentarios, atributos del útero, preocupaciones, y cambios en la sexualidad. Educar los hombres parejas de mujeres histerectomizadas es una acción fundamental para apoyar a las mujeres que serán sometidas a una histerectomía, siendo necesaria su incorporación en el plan de cuidados de ellas.

\section{DESCRIPTORES}

Histerectomía

Hombres

Sexualidad

Salud de la mujer

Investigación cualitativa

\section{RESUMO}

O objetivo deste estudo foi conhecer o significado da histerectomia para um grupo de homens chilenos, parceiros de mulheres histerectomizadas (HPMH). A pesquisa qualitativa foi realizada com entrevistas em profundidade, realizada com um total de quinze homens parceiros de mulheres histerectomizadas, entre maio e setembro de 2010, com aprovação prévia dos Comitês de Ética. Para a análise dos dados foi utilizada a perspectiva fenomenológica descrita por Giorgi e realizada a análise de conteúdo de acordo com Krippendorff. Os critérios de Crestwell foram utilizados para avaliar a credibilidade da análise e garantir a validez descritiva. Surgiram cinco dimensões que representam aspectos únicos do significado da extração do útero para os homens parceiros de mulheres histerectomizadas: sintomas, comentários, atribuições do útero, preocupações e mudanças na sexualidade. Educar os homens parceiros de mulheres histerectomizadas é fundamental para apoiar as mulheres que serão submetidas a uma histerectomia, sendo necessário incorporá-los no plano de cuidados das mulheres histerectomizadas.

\author{
DESCRITORES \\ Histerectomia \\ Homens \\ Sexualidade \\ Saúde da mulher \\ Pesquisa qualitativa
}

\begin{abstract}
The objective of this study was to understand the meaning of hysterectomy according to a group of Chilean men, partners of women who have undergone the procedure (MPWH). This qualitative study was performed with in-depth interviews. A total of 15 men, partners of women who have undergone hysterectomy, were interviewed between May and September of 2010, under the approval of the Ethics Committees. Data analysis was performed using the phenomenological perspective proposed by Giorgi, and content analysis was performed according to Krippendorff. The Crestwell criteria were used to evaluate the trustworthiness of the analysis and guarantee descriptive validity. Five dimensions emerged, which represented unique aspects of hysterectomy according to the men: symptoms, comments, the attributions of the uterus, concerns and changes in sexuality. It is essential to educate MPWH in terms of the support required by women undergoing hysterectomy. Therefore they should be included in the care plan designed for women undergoing hysterectomy.
\end{abstract}

\author{
DESCRIPTORS \\ Hysterectomy \\ Men \\ Sexuality \\ Women's health \\ Qualitative research
}

\footnotetext{
${ }^{1}$ PhD. Enfermera-Matrona. Profesora Asistente de la Escuela de Enfermería de la Pontificia Universidad Católica de Chile. Santiago, Chile. aarayagu@uc.cl ${ }^{2}$ PhD. Enfermera-Matrona. Profesora Asociada de la Escuela de Enfermería de la Pontificia Universidad Católica de Chile. Santiago, Chile. murrutis@uc.cl ${ }^{3}$ Enfermero-Matrón, Instructor Adjunto de la Escuela de Enfermería de la Pontificia Universidad Católica de Chile. Santiago, Chile. dsjara@uc.cl ${ }^{4}$ Médico. Profesor Asistente de La Escuela de Medicina de la Pontificia Universidad Católica de Chile. Santiago, Chile. ssilva@ssmso.cl ${ }^{5}$ Graduanda de Enfermería de la Escuela de Enfermería de la Pontificia Universidad Católica de Chile. Santiago, Chile. mulira@uc.cl ${ }^{6}$ Enfermera-Matrona, Instructor Adjunto de la Escuela de Enfermería de la Pontificia Universidad Católica de Chile. Santiago, Chile. ccflores@uc.cl
} 


\section{INTRODUÇÃO}

La histerectomía (HT) es una de las cirugías ginecológicas más frecuente a nivel mundial ${ }^{(1-2)}$, siendo la patología benigna del útero su principal causa( ${ }^{(3)}$. Existen escasas publicaciones de la percepción que los hombres, parejas de mujeres histerectomizadas (HPMH), tienen sobre el significado de la extracción del útero en sus parejas. Este tema no ha sido publicado en Latinoamérica; existiendo solo una publicación mexicana que aborda la visión sobre la HT, pero los hombres son parejas de mujeres no histerectomizadas.

Los hombres son reconocidos como un apoyo importante por las mujeres durante el proceso de ser histerectomizadas, especialmente al momento de tomar la decisión de operarse ${ }^{(4)}$. Sin embargo, ellos tratan de evitar conversar con sus parejas de las preocupaciones y de los sentimientos que esta cirugía les genera a ellos ${ }^{(5)}$. Esta falta de comunicación puede verse agravada con la falta de conocimiento que tienen sobre las patologías uterinas ${ }^{(6)}$ y la cirugía ${ }^{(7)}$.

El útero es un órgano significativo para las mujeres y la sociedad, ya que culturalmente se encuentra asociado a características como femineidad, sexualidad y a la capacidad reproductiva de éstas. Por ello, la pérdida de este órgano no sólo impacta a las mujeres histerectomizadas, sino que también a sus parejas ${ }^{(2)}$. La recuperación y adaptación sexual post-quirúrgica pueden verse afectadas por la perspectiva que los hombres tengan sobre el significado de la $\mathrm{HT}^{(8)}$.

El objetivo de este estudio es conocer la percepción del significado que pueda tener la extracción del útero en un grupo de hombres chilenos, parejas de mujeres histerectomizadas, con el propósito de entender el fenómeno desde la perspectiva masculina y planificar programas educativos orientados y sensibilizados hacia la mujer y su pareja.

\section{MÉTODO}

Estudio cualitativo, con entrevistas en profundidad, realizado en un total de 15 hombres parejas de mujeres histerectomizadas, cuyo criterio de inclusión fue: ser pareja de una mujer histerectomizada por patología benigna en los últimos 6 meses previos a la recolección de los datos. El estudio fue realizado en el Complejo Asistencial Dr. Sótero del Río (CASR) en Santiago, Chile, establecimiento perteneciente al sistema público de salud. En el sistema público de atención durante el año 2008 se realizaron un total de 11.920 histerectomías, lo que equivale a $32 \mathrm{mu}$ jeres histerectomizadas diariamente en nuestro país solo en este sector de la población. En la Región Metropolita- na, incluyendo Santiago, un total de 4.402 histerectomías fueron practicadas el mismo año, y específicamente el Servicio de Salud Metropolitano Sur-Oriente (SSMSO), al cual pertenece el CASR, reportó un total de 789 mujeres histerectomizadas.

La recolección de los datos fue realizada entre los meses de Mayo a Septiembre del 2010. Se invitaron a participar a todos los hombres parejas de mujeres que fueron histerectomizadas en ese periodo de tiempo. En la medida que cada mujer fue entregando los datos de contacto de sus parejas, un profesional hombre los contactó telefónicamente para invitarlos a participar. Los criterios de inclusión fueron ser la pareja declarada de una mujer sometida a una histerectomía de causa ginecológica benigna o maligna y el deseo de participar en este estudio; el criterio de exclusión fue haber sido pareja de una mujer sometida a una histerectomía por causa obstétrica. Una vez que accedieron a participar, se les citó a la entrevista. Las entrevistas fueron realizadas por un enfermeromatrón, parte del equipo investigador, que fue entrenado para este propósito, con el fin de facilitar el diálogo y la expresión de ideas y sentimientos ${ }^{(9)}$.

Posterior a la firma del consentimiento, se inició la entrevista en una sala privada destinada para este propósito, las que duraron en promedio 40 minutos y fueron grabadas completamente, para posteriormente ser transcritas de manera textual por personas entrenadas para esta actividad. El inicio de cada una de ellas estuvo direccionada por la pregunta: ¿Qué ha significado para usted que le hayan sacado el útero? Se realizaron entrevistas en profundidad logrando la saturación de los datos, en los cuales ningún nuevo significado y/o dimensión fue adquirido en las últimas tres entrevistas, en consecuencia todos los significados pudieron ser clasificados en las dimensiones ya existentes. Cabe señalar que, a pesar que la saturación de datos se logró a la novena entrevista, se decidió cumplir con número de entrevistas programadas de manera de asegurar la repetición de esos datos. La devolución de los resultados obtenidos se realizo con cada uno de los hombres que participaron en este estudio. Esta investigación contó con la aprobación de los Comités de Ética de la Escuela de Enfermería de la Pontificia Universidad Católica de Chile y del CASR.

Para el análisis de los datos se utilizó la perspectiva fenomenológica ${ }^{(10)}$ y se realizó el análisis de contenido ${ }^{(11)}$. Cuatro investigadores del equipo analizaron separadamente las transcripciones utilizando la siguiente metodología: lectura inicial de la transcripciones sin interpretación; segunda lectura para identificar los significados, implícitos o explícitos, de cada hombre, respaldando cada uno de ellos con una frase textual; luego una vez identi- 
ficados los significados se construyeron las dimensiones. Se realizó una lectura final de las transcripciones para rectificar las dimensiones y/o significados antes descritos. Se llegó a un consenso de las dimensiones y significados encontrados después de que cada investigador planteó su propuesta de análisis.

Criterios de rigurosidad ${ }^{(12)}$ fueron utilizados para evaluar la credibilidad del análisis y asegurar la validez descriptiva. Con este objetivo, después de ser analizadas las entrevistas en profundidad, se devolvieron las dimensiones y significados encontrados a cada uno de los hombres participantes en este estudio de manera de corroborar la validez de los datos obtenidos por el mismo profesional que los entrevistó. Junto con esto, las dimensiones y los significados fueron respaldados por una descripción detallada de cada uno de los relatos, permitiendo al lector establecer la utilidad y aplicabilidad de los resultados de este estudio. Finalmente, las dimensiones y significados fueron examinados por el investigador principal y tres investigadores expertos en el área, quienes consideraron válidos los resultados obtenidos.

\section{RESULTADOS}

Con respecto a las características socio-demográficas, la mediana de edad fue de 46 años, con un rango entre 34 y 55 años. En relación al nivel educacional, 1 de ellos tiene enseñanza básica competa ( 8 años de escolaridad); 8 enseñanza media incompleta (entre 8 y 11 años), 3 enseñanza media completa (12 años) y 1 con enseñanza superior (más de 12 años). La mediana de convivencia en pareja fue de 22 años, con un rango de 10 a 35 años.

Del análisis de las entrevistas de los hombres parejas de mujeres histerectomizadas, emergieron cinco dimensiones que representan aspectos únicos del significado de la extracción del útero para sus parejas. En lo Cuadro 1 se encuentra la descripción de las éstas y sus respectivos significados extraídos de los relatos de los hombres.

Cuadro 1 - Dimensiones y significados extraídos de los relatos de los hombres, parejas de mujeres histerectomizadas $(n=15)$

\begin{tabular}{|l|l|}
\hline Dimensión & Significados \\
\hline \multirow{2}{*}{ Síntomas } & Tipos de síntomas pre-quirúrgicos \\
\cline { 2 - 2 } & Cese de los síntomas post-histerectomía \\
\hline \multirow{2}{*}{ Comentarios } & Quedar Hueca/Vacía \\
\cline { 2 - 2 } Atributos del útero & Sexualidad después de la histerectomía \\
\hline \multirow{2}{*}{ Preocupaciones } & No tener hijos \\
\cline { 2 - 2 } & Elgo íntimo \\
\cline { 2 - 2 } & Esfera sexual \\
\cline { 2 - 2 } Cambios en la sexualidad \\
\hline & Cáncer \\
\cline { 2 - 2 } & Autoevaluación positiva de la sexualidad \\
\hline & Autoevaluación negativa de la sexualidad \\
\hline
\end{tabular}

La dimensión síntomas involucra las señales físicas visibles causadas por una patología ginecológica que son detectadas por los hombres en su pareja antes de la HT, junto con la identificación de cambios producto del cese de los mismos. El síntoma más señalado por los hombres son el sangramiento abundante durante el período prequirúrgico.

Hombre: ...tenía unos sangramientos cototos, yo te digo 15 días, 18 días, 22 días de repente de sangramiento, era una cuestión que nos tenía enfermos a nosotros a los dos en general porque... ella se sentía mal...

Hombre: $Y$ normalmente eh... sus reglas eran muy abundantes, eh... muy dolorosas, muy largas entonces eso era lo más que le complicaba después de... dentro de los cuatro años ya los últimos años era caótico.

Hombre: ... la cama manchada donde botaba sangre, si con decirte que a veces estaba dos, tres días pero botaba, botaba, botaba... y todavía está...y empapada, toda la noche, entonces... ahora ya no ya, ahora estamos contentos, yo estoy contento, y ella también se siente bien, ya tiene otra cara...

La HT es una cirugía que está rodeada de mitos y creencias que los hombres escuchan y que identificaron en la dimensión comentarios. En general estos comentarios tienen una connotación negativa de la cirugía. Los significados identificados en esta dimensión fueron quedar vacía o hueca y sobre la sexualidad después de la HT.

Hombre: Claro, y a veces entre hombres dicen...oye pero generalmente las mujeres quedan huecas po' te vas a tener que buscar otra.

Hombre: ...Porque ella antes de la operación sufría ella, sufría yo, después que no se quería operar por el motivo que todos decían que iba a quedar el... la palabra a lo chileno, hueca...eeh que me iba a afectar a mí en la relación sexual, que yo a lo mejor no la iba a quererla ehhh muchas cosas...

Hombre: ... igual teníamos algunos prejuicios de esto porque mucha gente opina y uno igual tiene sus preocupaciones porque decían de que las hormonas y todas esas cosas de que iba que ella después prácticamente no iba a sentir placer ella cuando después de la operación.

El atributo del útero es una dimensión que refleja las cualidades que se le otorgan al útero desde la perspectiva masculina. Se destaca la pérdida de la capacidad reproductiva y la extracción del útero como una experiencia privada, íntima, que la pareja (ambos) sólo comparten con personas de confianza.

Hombre: Lloramos en conjunto con la cuestión porque... no sé es una cosa de tu cuerpo que te la saquen y saber que por el intermedio de esto viene una reproducción que fue... entonces le decía yo a mi señora miremos a nuestros hijos y nos recordamos que... que nacieron de ahí no más'...

Hombre: ... lo que más desea una mujer es tener hijos tener todo su cuerpo intacto para poder criar tener un hijo
El significado de la histerectomía para un grupo de hombres chilenos parejas de histerectomizadas Gutiérrez AA, Soto MTU, Suazo DJ, Solovera SS, Salas MJL, Espinoza CF 
y qué se yo, tú sabes que el hijo es lo más bonito que hay en la vida pero eso es lo más lo más bueno que puede suceder en este caso a una mujer y a uno también como hombre es algo bueno.

Hombre: siempre lo mantuvimos entre nos, creo que... es algo entre pareja y nosotros lo estimamos que no tenia porqué ir más allá de un hermano, una cuñada, una concuñada...

La dimensión preocupaciones describe como los hombres experimentan intranquilidad, inquietud y/o temor a la cirugía, a la eventualidad de un pronóstico negativo como el cáncer o al funcionamiento sexual de su pareja.

Hombre: Preocupado por ella, por la salud de que... que pensar de que a lo mejor no iba, no iba a quedar bien o podía tener alguna consecuencia después de la operación... eso.

Hombre: uno entremedio escucha tantas cosas y uno entra a desesperarse porque uno piensa que a su mujer le va a dar un cáncer y tantas cosas que enfermedades que están dando...

Hombre: Que ella no iba a sentir orgasmos yo eso me tenía preocupado porque eh eso es importante y no hemos tenido problema todo lo contrario...

La dimensión cambios en la sexualidad involucra aquellos relatos en los cuales los hombres hacen una autoevaluación de los comportamientos y conductas relacionadas con la actividad sexual antes de la HT y a los cambios posteriores a la cirugía.

Hombre: Se pierde la, la parte sexual digamos te jode automáticamente o sea eso es obvio, le cortan la luz y el agua a uno y eh pero después vuelve sin problema...

Hombre: Sí, no muy buena, muy buena y mejoró como le digo, lo más importante del matrimonio de la pareja, mejoró en sentido de lo sexual en todo...Pal' hombre mismo.

Hombre: desde que se operó hasta ahora hemos tenido solamente una sola vez hemos... hemos tenido relaciones sexuales, ella ha tenido inconscientemente o conscientemente no sé... una inapetencia sexual y es una incomodidad de ella cuando uno se acerca... mmm según ella dice que se siente bien pero en realidad yo noto que no...

\section{DISCUSIÓN}

Existen distintos estudios que explican el impacto de la $\mathrm{HT}$ en la vida de las mujeres, siendo la perspectiva masculina la menos abordada. Este es uno de los pocos estudios publicados acerca del significado de la extracción del útero en un grupo de HPMH y es el primero en describir la experiencia en un grupo de hombres chilenos.

Las molestias ginecológicas tienen un gran impacto en la salud de las mujeres ${ }^{(13)}$, afectando negativamente su calidad de vida ${ }^{(6)}$ y limitando sus actividades cotidia- nas ${ }^{(14)}$. En este estudio, los síntomas más reconocidos por los hombres fueron la hemorragia y síntomas de anemia lo que concuerda con la literatura internacional ${ }^{(6,13,15)}$. La sensación de alivio de los síntomas que desencadenaron la cirugía es una de los significados más reportadas por los hombres parejas de mujeres histerectomizadas ${ }^{(1,4)}$. Los hombres identificaron que los síntomas previos a la cirugía han afectado negativamente su relación de pareja; y por ende, la cirugía es vista como una forma de tratar dichos síntomas. Este hallazgo concuerda con lo reportado por otros investigadores tanto en el alivio de los síntomas ${ }^{(15)}$, como en la percepción de mejoría del estado de salud de la mujer ${ }^{(13)} y$ de su vida sexual ${ }^{(1,4)}$.

Los comentarios fueron otro de los resultados de este estudio, siendo una de las dimensiones que más daño puede causar tanto a la mujer como a la relación de pareja. Quedar hueca o vacía fue una de los significados que emergieron en la dimensión de significados. En este sentido la literatura señala que tener una HT ha sido asociada como una experiencia negativa para ambos miembros de la relación ${ }^{(2,4,7)}$. Las mujeres identifican que los hombres se refieren a las mujeres histerectomizadas en términos negativos $^{(4,16)}$. A pesar que ellas puedan identificar que estos comentarios nacen del desconocimiento de los hombres sobre la cirugía, las palabras pueden tener el poder de socavar su autoestima ${ }^{(4)}$. Con respecto al segundo significado identificado por los hombres, comentarios a cerca de la sexualidad después de la HT, la literatura señala que las mujeres, cuyas parejas tienen una actitud negativa relacionada a la incapacidad para tener hijos o a la posible alteración en la sexualidad después de la HT, piensan que la cirugía puede afectar su relación de pareja e incluso podría llegar al término de ésta(4). En algunos casos, los comentarios negativos sobre la cirugía vienen de mujeres y hombres que no han vivido el proceso de una HT en su mujer, siendo éstas las que tienen una peor percepción sobre la cirugía ${ }^{(2)}$. Ni los hombres ni las mujeres tienen conocimientos apropiados sobres sus cuerpos; por lo que pueden desarrollan percepciones incorrectas sobre ellos y las funciones del mismo ${ }^{(7)}$.

En este estudio, los atributos del útero identificados por los hombres fueron el no tener hijos y que es algo íntimo. Investigadores señalan que los atributos del útero están relacionados al valor que entrega el hombre a la pérdida de la capacidad reproductiva femenina ${ }^{(1,2)}$. Culturalmente el rol de la mujer está asociado a su posibilidad de engendrar, siendo la maternidad uno de los aspectos más reconocidos socialmente ${ }^{(17)}$. Por otro lado, los hombres parejas de mujeres histerectomizadas identificaron que la extracción del útero es una experiencia íntima, que se vive dentro de la pareja y que solo se comparte con su núcleo familiar más cercano. Esta privacidad de la experiencia puede verse explicada por los mitos y creencias que esta cirugía tiene socialmente y de esta manera la privacidad es una forma de protegerse frente a la opinión de terceros, la cual resulta ser negativa. 
Los hombres parejas de mujeres histerectomizadas manifestaron preocupaciones en la esfera sexual y miedo a que sus miedo a que sus parejas tuvieran cáncer, resultados que concuerdan con otros estudios en el sentido que los hombres conocen muy poco sobre las consecuencias de la cirugía y manifiestan temores y preocupaciones sobre sus consecuencias a corto y largo plazo ${ }^{(1,6)}$. Por ejemplo, los hombres piensan que el útero es un órgano de mayor tamaño de lo que es en realidad, lo que se correlaciona con el miedo a la cirugía(1). También los hombres manifiestan miedo a que las mujeres puedan desarrollar una patología maligna producto de las causas que generaron la $\mathrm{HT}^{(1,6,7)}$, principalmente relacionado con el resultado de la biopsia. Las preocupaciones en la esfera sexual es una de las inquietudes más frecuentes del género masculino ${ }^{(5)}$, que también es compartida por las mujeres $^{(6,15)}$. Ellas reportan frecuentemente inquietudes de sus parejas tales como: miedo a que las relaciones sexuales no sean buenas o miedo a que sus parejas sean menos mujeres después de la cirugía(4). En este sentido, hombres de Noruega reportan satisfacción con sus erecciones y un mejoramiento de su vida sexual después de la $\mathrm{HT}^{(18)}$, desmitificando la idea que las mujeres histerectomizadas no los pueden satisfacer sexualmente. En la misma línea, mujeres afroamericanas, reportan que la mayoría de las hombres no pueden darse cuenta que el útero fue removido durante la actividad sexual ${ }^{(4)}$.

En relación a los autoevaluación de la sexualidad, los hombres en este estudio identificaron cambios positivos y negativos. Evidencia científica nacional señala que la sexualidad definitiva dependerá en gran medida de cómo era su sexualidad y la comunicación de pareja previo a la $\mathrm{HT}^{(19)}$. Lo que concuerda con lo reportado por estudios internacionales donde mujeres ${ }^{(20)}$ y hombres ${ }^{(7,18)}$ señalan tener la misma o una mejor vida sexual de la que tenían antes de la cirugía.

El significado de la HT se ve explicado por las dimensiones de síntomas, comentarios, atributos del útero, preocupaciones y cambios en la sexualidad. Una atención integral

\section{REFERENCIAS}

1. Lalos A, Lalos O. The partner's view about hysterectomy. J Psychosom Obstet Gynaecol. 1996;17(2):119-24.

2. Maraván ML, Trujillo $P$, Karam MA. Hysterectomy as viewed by Mexican women and men. Sex Roles. 2009;61(9-10):688-98.

3. Kramer MG, Reiter RC. Hysterectomy: indications, alternatives and predictors. Am Fam Physician. 1997;55(3):827-34.

4. Richter DL, McKeown RE, Corwin SJ, Rheaume C, Fraser J. The role of male partners in women's decision making regarding hysterectomy. J Womens Health Gend Based Med. 2000;9 Suppl 2:S51-61. en salud basada en una interacción mutua entre profesionales de la salud y usuarios ${ }^{(21)}$ y abordando las necesidades educativas de las mujeres y sus parejas ${ }^{(22-23)}$ son una pieza fundamental de abordar en la atención de salud.

\section{CONCLUSIÓN}

Los resultados de este estudio nos muestran que la extracción del útero tiene un impacto en las parejas de las mujeres histerectomizadas. Este impacto se refleja no solo en variables clínicas, como el alivio de la sintomatología postquirúrgica, sino que también en variables como comentarios, atributos del útero, preocupaciones y cambios en la sexualidad que colocan en evidencia que la histerectomía es un procedimiento multidimensional involucrando variables físicas, psicológicas y sociales.

Es necesario contar con programas educativos adecuados a las necesidades del hombre y de su pareja previa y posterior a la cirugía. El apoyo más significativo para una mujer histerectomizada es el de su pareja, buscando en él soporte emocional y consuelo, influyendo incluso en su recuperación postoperatoria. Es importante educar a los hombres es una pieza fundamental para apoyar a las mujeres que serán sometida a una histerectomía; ya que estos tienen poco conocimiento sobre esta cirugía, manifestándose en el escaso dominio sobre el procedimiento quirúrgico, el desconocimiento sobre las adaptaciones en la vida sexual posterior a la cirugía y en el proceso de la toma de decisión sobre la histerectomía.

Existe una necesidad de entregar educación e información oportuna a los HPMH y sus parejas. Esta necesidad educativa se transforma en una oportunidad única para que los profesionales de la salud y, específicamente, para las enfermeras, establezcan programas educativos, centrados en las necesidades de las parejas y que se integren al plan de cuidados de las mujeres en proceso de ser histerectomizadas, siendo la incorporación de los hombres un pieza fundamental y una tarea pendiente.

5. Bernhard LA, Harris CR, Caroline HA. Partner communication about hysterectomy. Health Care Women Int. 1997;18(1):73-83.

6. Chou CC, Lee TY, Sun CC, Lin SS, Chen LF. Husbands' experiences before wives' hysterectomy. J Nurs Res. 2006;14(2):113-22.

7. Bernhard LA. Men's views about hysterectomies and women who have them. Image J Nurs Sch. 1992;24(3):177-82.

8. Newman G, Newman LE. Coping with the stress of hysterectomy. J Sex Educ Ther. 1985;11(2):65-8.

9. Broom A, Hand K, Tovey P. The role of gender, environment and individual biography in shaping qualitative interview data. Int J Soc Res Methodol. 2009;12(1):51-65. 
10. Giorgi A. Sketch of a psychological phenomenological method. In: Aanstoos CM, Fischer WF, Giorgi A, Wertz FJ, editors. Phenomenology and psychological research. Pittsburgh: Duquesne University Press; 1985.

11. Krippendorff K. Content analysis: an Introduction to its methodology. Thousand Oaks: Sage; 2003.

12. Creswell JW. Standards of validation and evaluation: qualitative inquiry and research design: choosing among five approaches. 2nd ed. Thousand Oaks: Sage; 2007. p. 201-21.

13. Rannestad T, Eikeland OJ, Helland H, Qvarnstrom U. The general health in women suffering from gynaecological disorders is improved by means of hysterectomy. Scand J Caring Sci. 2001;15(3):264-70.

14. Reis N, Engin R, Ingec M, Bag B. A qualitative study: beliefs and attitudes of women undergoing abdominal hysterectomy in Turkey. Int J Gynecol Cancer. 2008;18(5):921-28.

15. Lindberg CE, Nolan LB. Women's decision making regarding hysterectomy. J Obstet Gynecol Neonatal Nurs. 2001;30(6):607-16.

16. Marván ML, Islas M, Vela L, Chrisler JC, Warren EA. Stereotypes of women in different stages of their reproductive life: data from Mexico and the United States. Health Care Women Int. 2008;29(7):673-87.
17. Salvador RT, Vargens OMC, Progianti JM. Sexualidade e histerectomia: mitos e relidade. Rev Gaúcha Enferm. 2008; 29(2):320-3.

18. Lonnee-Hoffmann RA, Schei B, Eriksson NH. Sexual experience of partners after hysterectomy, comparing subtotal with total abdominal hysterectomy. Acta Obstet Gynecol Scand. 2006;85(11):1389-94.

19. Urrutia MT, Araya A, Rivera S, Viviani P, Villarroel L. Sexualidad de la mujer histerectomizada: modelo predictivo al sexto mes posterior a la cirugía. Rev Med Chil. 2007;135(3):317-25.

20. Helstrom L, Sorbom D, Backstrom T. Influence of partner relationship on sexuality after subtotal hysterectomy. Acta Obstet Gynecol Scand. 1995;74(2):142-6.

21. Fracolli LA, Zoboli ELP, Granja GF, Ermel RC. The concept and practice of comprehensiveness in Primary Health Care: nurses' perception. Rev Esc Enferm USP [Internet]. 2011 [cited 2011 Nov 25];45(5):1135-41. Available from: http://www. scielo.br/pdf/reeusp/v45n5/en_v45n5a15.pdf

22. Araya A, Urrutia MT, Muñoz L, Villa L. El proceso de "ser histerectomizada": Aspectos educativos a considerar. Rev Chil Obstet Ginecol. 2008;73(5):347-52.

23. Urrutia MT, Riquelme P, Araya A. Educación de mujeres histerectomizadas: ¿Qué desean saber? Rev Chil Obstet Ginecol. 2006;71(3):410-16. 